\title{
RISK PREDICTION FOR NIEMANN-PICK DISEASE
}

A retrospective chart review of 216 patients with Niemann-Pick disease type C (NP-C) was conducted in 5 centers in Europe including University of Amsterdam and 2 in Australia. Three patient types were selected: classic or variant filipin staining NP-C cases $(n=71)$ including family members with NP-C, NP-C filipin-negative staining noncases $(n=64)$, or controls with at least 1 characteristic symptom of NP-C $(n=81)$. NP$\mathrm{C}$ symptoms and signs were categorized into visceral, neurologic, or psychiatric domains. Logistic regression was performed on individual signs and symptoms within and across domains, and regression coefficients were used to develop prediction scores for NP-C.

The suspicion index tool has good discriminatory performance, and patients with a score $>70$ should be tested for NP-C. Strong predictors of NP-C are neonatal jaundice/cholestasis, splenomegaly, vertical supranuclear gaze palsy, cataplexy, and cognitive decline/dementia; also, symptoms occurring in multiple domains in individual patients, and parents/siblings or cousins with NP-C. (Wijburg FA, Sedel F, Pineda M, et al. Development of a suspicion index to aid diagnosis of Niemann-Pick disease type C. Neurology May 15;78(20):1560-1567). (Response and reprints: Dr Wijburg. E-mail: f.a.wijburg@amc.uva.nl).

COMMENT. NP-C is a rare inherited neurovisceral disease caused by mutations in the NPC1 $(95 \%)$ or NPC2 gene (5\% cases) that lead to accumulation of cholesterol and glycosphingolipids in the brain, liver and other tissues. Foamy cells are present in the bone marrow, spleen and liver, and sea-blue histiocytes in the bone marrow. Neurological manifestations include saccadic eye movement abnormalities or vertical supranuclear gaze palsy, cerebellar ataxia, dystonia, dysmetria, dysarthria and dysphagia, gelastic cataplexy, and seizures. Age at presentation is early-infantile, late infantile, juvenile or adolescent/adult. International guidelines for management of NP-C were published in 2009, updated 2011. Disease-specific therapy with miglustat is reevaluated. (Patterson MC et al. Mol Genet Metab 2012 May 7. Epub ahead of print).

Four varieties of NP disease are distinguished, ABC \& D. Type A is the classic infantile neuronopathic form, presenting with failure to thrive, persistent neonatal jaundice, hepatomegaly, lymphadenopathy, and sometimes a retinal cherry red spot. It is more common in Ashkenazi Jewish families. Type D is found in Nova Scotian families.

\section{AUTOIMMUNE AND DEMYELINATING DISORDERS}

\section{MYASTHENIA GRAVIS AND NEUROMYELITIS OPTICA ASSOCIATION}

Investigators at the University of Oxford, UK and 8 other international neurology centers describe the clinical, serological, and temporal associations of myasthenia gravis (MG) and neuromyelitis optica spectrum disorder (NMOSD) in 16 patients. All had early onset acetylcholine receptor antibody [AChR-Ab]-mediated $\mathrm{MG}$, the majority with mild generalized disease, and a high proportion achieved remission. The MG preceded 
NMOSD by a median of 16 years, and 11 had thymectomy. Aquaporin- 4 antibodies [AQP4-Ab] were detectable between 4 and 16 years prior to NMOSD onset. AChR-Abs decreased and the AQP4-Ab levels increased over time in concordance with MG and NMOSD, respectively. AChR-Abs were detectable at NMOSD onset in one of 2 patients diagnosed with NMOSD before MG. (Leite MI, Coutinho E, Lana-Peixoto M, et al. Myasthenia gravis and neuromyelitis optica spectrum disorder. A multicenter study of 16 patients. Neurology 2012 May 15;78:1601-1607). (Response and reprints: Dr Palace. Email: Jacqueline.palace@clneuro.ox.ac.uk).

COMMENT. The association of $\mathrm{MG}$ and NMOSD, 2 rare organ-specific autoimmune diseases mediated by 2 distinct antibodies, occurs more frequently than by chance. MG usually presents first, and respective antibodies are present years before onset of the relevant disease. The $\mathrm{MG}$ tends to be relatively mild and treatment responsive. Recent evidence suggests that the thymus is involved in both $\mathrm{MG}$ and NMOSD (Chan KH et al. J Neuroimmunol 2010;227:178-184; cited by authors). Given the risk of concurrent autoimmune diseases in patients with $\mathrm{MG}$ or NMO, routine evaluation of thyroid antibodies and AQP4-Abs may be considered in patients with early onset AChR-MG.

\section{PROTEOMIC TECHNIQUES AS BIOMARKERS FOR MULTIPLE SCLEROSIS}

Researchers at the Montreal Neurological Institute and other centers in Canada performed proteomics screening of CSF samples collected from 19 children at presentation of acquired inflammatory CNS demyelinating syndromes. Children were followed prospectively and 8 developed MS-defining recurrent disease activity (acquired CNS demyelinating syndrome [ADS]); 11 had no recurrent disease (ADS-monophasic) over a median period of 4.88 years (range, 2.52-6.12 years). Mass spectroscopy, peptide profiling, and quantitative immunoblotting were used to identify CSF proteins that might discriminate MS from monophasic demyelination.

Major compact myelin membrane proteins typically implicated in MS were not detected. Instead, multiple molecules that localize to the node of Ranvier and the surrounding axoglial apparatus membrane were increased by 10.2 -fold in children subsequently diagnosed with MS. The CSF proteome signature obtained at the presentation of CNS inflammation may be predictive of subsequent MS diagnosis. (Dhaunchak AS, Becker C, Schulman H, et al on behalf of the Canadian Pediatric Demyelinating Disease Group. Ann Neurol 2012 May;71:601-613). (Respond: Dr Amit Bar-Or, Neuroimmunology, Montreal Neurological Institute, McGill University, 3801 University Street, Room 111, Montreal, Quebec, H3A284, Canada. E-mail: amit.baror@mcgill.ca).

COMMENT. In the same issue of the Annals, investigators from Japan report on the use of CSF proteomic pattern analysis to discriminate MS-related disorders in 107 adult patients. (Komori $M$ et al. Ann Neurol 2012 May;71:614-623). An editorial (Bennett JL Owens GP. Ann Neurol 2012 May;71:587-588) comments that CSF proteomics is a promising window and biomarker for demyelinating disorders. 